\title{
Primary peripheral nerve sheath tumors of the thyroid gland: A case report and literature review
}

\author{
GUANG CHEN $^{1 *}$, ZENGGUANG LIU $^{1 *}$, CHANG SU $^{1}$, QIANG GUAN ${ }^{2}$, FANG WAN $^{1}$, BINGFEI DONG ${ }^{1}$, \\ LIANG BAO $^{1}$, WENXIN ZHANG ${ }^{1}$, YINPING WANG ${ }^{3}$ and GUIMIN WANG ${ }^{1}$ \\ ${ }^{1}$ Department of Thyroid Surgery, The First Hospital of Jilin University, Changchun, Jilin 130021; \\ ${ }^{2}$ Department of General Surgery, Changchun Central Hospital, Changchun, Jilin 130051; \\ ${ }^{3}$ Department of Pathology, The First Hospital of Jilin University, Changchun, Jilin 130021, P.R. China
}

Received May 7, 2015; Accepted August 4, 2015

DOI: $10.3892 /$ mco.2015.703

\begin{abstract}
Primary peripheral nerve sheath tumors (PNSTs) of the thyroid gland are rare, with fewer than 30 cases reported in the medical literature to date. Primary PNSTs of the thyroid gland are classified into malignant and benign PNSTs. The benign PNSTs may be further subclassified into neurofibromas and Schwannomas. This is the case report of a 51-year-old male patient presenting with multiple primary PNSTs involving the left lobe of the thyroid gland. The patient underwent total excision of the thyroid gland and the pathological results indicated a Schwannoma with Antoni type A and B cells. The literature was reviewed briefly and, to the best of our knowledge, this is the first case report of multiple primary PNSTs of the thyroid gland.
\end{abstract}

\section{Introduction}

Primary peripheral nerve sheath tumors (PNSTs) of the thyroid gland are rare, accounting for $<0.02 \%$ of all thyroid tumors worldwide (1). PNSTs may occur at any age, with the highest incidence between 40 and 60 years of age, without notable between-gender differences. PNST usually presents as a gradually enlarging mass of the thyroid, with non-specific symptoms and signs, which makes preoperative diagnosis difficult; curative surgery is currently the mainstay of treatment. PNSTs may be classified as benign or malignant (MPNSTs) and they often originate from anatomically discernible peripheral nerves. MPNSTs mostly occur between the ages of 20 and 50 years, presenting as asymptomatic nodules, or with symp-

Correspondence to: Professor Guimin Wang, Department of Thyroid Surgery, The First Hospital of Jilin University, 71 Xinmin Avenue, Chaoyang, Changchun, Jilin 130021, P.R. China E-mail: 493090494@qq.com

${ }^{*}$ Contributed equally

Key words: thyroid gland, peripheral nerve sheath tumor, Schwannoma toms including difficulty in breathing and weight loss, which are associated with a poor prognosis $(2,3)$.

\section{Case report}

A 51-year-old man presented with a 4-year history of a slowly growing thyroid mass, without any history of dysphagia, hoarseness or weight loss. The physical examination revealed a non-tender, mobile nodule in the left lobe of the thyroid gland, $\sim 30 \times 20 \mathrm{~mm}$ in size with well-defined margins. Cervical lymphadenopathy was clinically absent and all the laboratory tests, including fT3, T3, fT4, T4, thyroid-stimulating hormone and calcitonin were within the normal range. Ultrasonography revealed two adjacent, well-defined, hypoechogenic solid nodules within the left lobe of the thyroid gland, sized $25.4 \times 15.5$ and $14.4 \times 9.1 \mathrm{~mm}$ (Fig. 2). Total excision of the thyroid gland was performed. A detailed pathological examination was conducted and the final diagnosis was benign PNSTs of the left lobe of the thyroid gland. To date, the patient has been followed up for 6 months and his thyroid hormone profile, other laboratory tests and thyroid ultrasonography findings are normal.

\section{Discussion}

Primary PNSTs of the thyroid gland are classified into MPNSTs and benign PNSTs. The benign PNSTs may be further subclassified into neurofibromas and Schwannomas.

Neurofibromas mainly arise from peripheral or cutaneous nerves, and usually occur sporadically, although they may coexist with neurofibromatosis. Neurofibromas exhibit biallelic inactivation of the NFI gene, which encodes the neurofibromin protein (4-6).

Schwannomas originate from neuronal sheath cells (also known as Schwann cells), and are usually slow-growing tumors presenting in the fourth to sixth decades of life. Schwannomas may lead to pressure symptoms resulting from direct compression of adjacent organs, such as the thyroid gland (7). As previously described, Schwannomas are grouped into two histological types: Type A is characterized by pallisading and spindle-shaped Schwann cells; and type B usually exhibits a sparsely cellular pattern with cystic degeneration (8). 


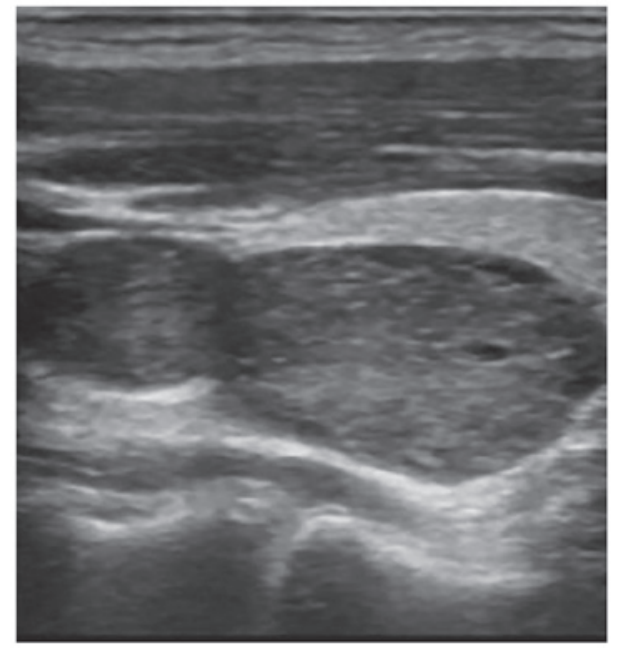

Figure 1. Ultrasonography of the thyroid gland revealing two solid hypoechoic mass lesions in the left lobe.

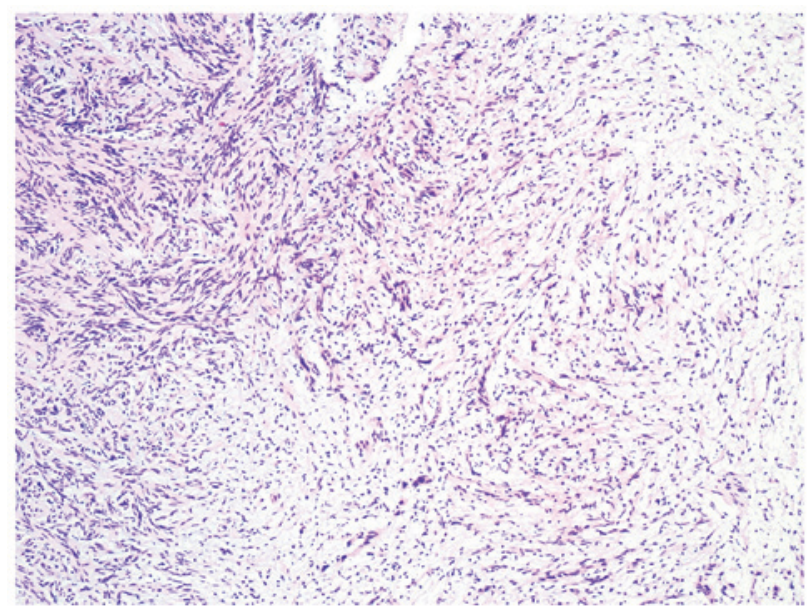

Figure 2. Hematoxylin and eosin staining of peripheral nerve sheath tumor tissue (magnification, $\mathrm{x} 40$ ). Upper left corner of the figure, area with spindle-shaped Schwann cells (Antoni type A); bottom right corner of the figure, area with scattered cells with a few unorganized nuclei (Antoni type B).

The majority of patients with Schwannomas of the thyroid gland only present with a painless, slowly-growing mass, without any other symptoms (3). The ultrasonography and computed tomography (CT) scans usually reveal a well-delineated, solid nodule, wihtout involvement of the cervical lymph nodes $(3,8)$. The laboratory results are usually within the normal range (3). It has been reported in one case that fine-needle aspiration was helpful in reaching a definitive diagnosis (9).

In the clinical setting, benign PNSTs should be carefully differentiated from MPNSTs. MPNSTs are a group of invasive tumors, which may result in a fatal outcome irrespective of administering aggressive adjuvant therapies $(3,10)$. MPNSTs may efface the thyroid parenchyma in a fascicular pattern of growth; they are characterized by neural-appearing cells, increased cellularity, increased mitotic activity and focal necrosis (3). The immunohistochemical staining for S-100, CD34 and vimentin may be helpful in confirming the diagnosis (3).
Since the majority of the PNSTs of the thyroid gland are asymptomatic and fine-needle aspiration is not very efficient, it is difficult to reach a definitive a diagnosis prior to surgical removal $(11,12)$. To the best of our knowledge, the reported outcomes of these patients are satisfactory, with a low recurrence rate (11-13).

In conclusion, PNSTs of the thyroid gland are rare and generally asymptomatic. Although the majority of these tumors are benign, surgical resection is required for the final diagnosis and is the main therapeutic option for symptomatic patients.

\section{References}

1. Thompson LD, Wenig BM, Adair CF and Heffess CS: Peripheral nerve sheath tumors of the thyroid gland: A series of four cases and a review of the literature. Endocr Pathol 7: 309-318, 1996.

2. Kandil E, Abdel Khalek M, Abdullah O, Dali D, Faruqui S, Khan A, Friedlander P, Jaffe BM and Crawford B: Primary peripheral nerve sheath tumors of the thyroid gland. Thyroid 20: 583-586, 2010.

3. Pallares J, Perez-Ruiz L, Ros S, Panades MJ, Pardo-Mindan J, Lloreta J and Matias-Guiu X: Malignant peripheral nerve sheath tumor of the thyroid: A clinicopathological and ultrastructural study of one case. Endocr Pathol 15: 167-174, 2004.

4. Baglaj M1, Markowska-Woyciechowska A, Sawicz-Birkowska K and Dorobisz U: Primary neurilemmoma of the thygland in a 12-year-old girl. J Pediatr Surg 39: 1418-1420, 2004.

5. Aoki T, Kumeda S, Iwasa T, Inokawa K, Hori T and Makiuchi M: Primary neurilemoma of the thyroid gland: Report of a case. Surg Today 23: 265-268, 1993.

6. Delaney WE and Fry KE: Neurilemoma of the thyroid gland. Ann Surg 160: 1014-1017, 1964.

7. Graceffa G, Cipolla C, Florena AM, Gentile I, Pompei G and Latteri MA: Primary schwannoma of the thyroid gland involving the isthmus: Report of a case. Surg Today 43: 106-109, 2013.

8. Sugita R, Nomura T and Yuda F: Primary schwannoma of the thyroid gland: CT findings. AJR Am J Roentgenol 171: 528-529, 1998.

9. Jayaram G: Neurilemmoma (schwannoma) of the thyroid diagnosed by fine needle aspiration cytology. Acta Cytol 43: 743-744, 1999.

10. Hruban RH, Shiu MH, Senie RT and Woodruff JM: Malignant peripheral nerve sheath tumors of the buttock and lower extremity. A study of 43 cases. Cancer 66: 1253-1265, 1990.

11. Kang GC, Soo KC and Lim DT: Extracranial non-vestibular head and neck schwannomas: A ten-year experience. Ann Acad Med Singapore 36: 233-238, 2007.

12. Kar M, Deo SV, Shukla NK, Malik A, DattaGupta S, Mohanti BK and Thulkar S: Malignant peripheral nerve sheath tumors (MPNST) - clinicopathological study and treatment outcome of twenty-four cases. World J Surg Oncol 4: 55-62, 2006.

13. Gustafson LM, Liu JH, Rutter MJ, Stern Y and Cotton RT: Primary neurilemoma of the thyroid gland: A case report. Am J Otolaryngol 22: 84-86, 2001. 